\title{
Cunf-9205147--5i:
}

To be published in Proc. Tenth Symposium of Energy Sciences, held at the Argonne National Laboratory, Chicago, II, May 11 - 13, 1992.

\section{COHERENCE EFFECTS IN RADIOMETRY AND IN SPECTROSCOPY}

\author{
Emil Wolf* \\ Department of Physics and Astronomy, University of Rochester \\ Rochester, New York 14627
}

\begin{abstract}
Some recent researches are described concerning the effects of the state of coherence of a source on the spatial and the spectral distributions of energy generated by the source. The researches have elucidated the foundations of radiometry and they have also revealed some unexpected new phenomena relating to spectral changes which can be induced by source correlations and also by scattering on random media.
\end{abstract}

\section{INTRODUCTION}

Until 1960 practically all known sources of optical radiation were thermal sources, such as incandescent matter or gas discharge. The situation has changed drastically in 1960 when the first lasers were made. From the standpoint of statistical physics these two types of sources represent two extreme situations. Thermal sources are highly uncorrelated (incoherent), whereas well-stabilized single-mode lasers are completely correlated (fully coherent). Today sources of many other kinds are available, whose statistical properties are intermediate between these two extremes but they are not as well understood as thermal sources and lasers. What is quite clear, however, is that the statistical properties of a source may affect drastically the nature of the radiation field which the source generates. Our research has been mainly concerned with elucidating how some of the basic statistical features of a source, more specifically its socalled second-order coherence properties, affect the energy distribution in the field generated by the source.

\section{SECOND-ORDER CORRELATION FUNCTIONS}

Let me first recall what is meant by second-order coherence properties of sources and of fields. To explain the essential features I will ignore polarization properties and I will treat the source variable and the field variable as scalar quantities. Let $Q(r, t)$ be the source variable and $V(r, t)$ the field variable, representing the fluctuating source and the fluctuating field respectively at a point $r$, at time $t$. We use the so-called complex analytic signal representation of these quantities ${ }^{1}$.

We assume that the fluctuations are statistically stationary, at least in the wide sense. The secondorder coherence properties of the source and of the field may then be characterized by the cross-correlation functions

$$
\Gamma_{Q}\left(r_{1}, r_{2}, \tau\right)=\left\langle Q^{*}\left(r_{1}, t\right) Q\left(r_{2}, t+\tau\right)\right), \quad \Gamma_{V}\left(r_{1}, r_{2}, \tau\right)=\left\langle V^{*}\left(r_{1}, t\right) V\left(r_{2}, t+\tau\right)\right\rangle
$$

where the asterisks denotes the complex conjugate and the angle brackets denote the ensemble average. For our purposes it will be more convenient to characterize the second-order coherence properties of the

DISTRIBUTION OF THIS DOCUMENT IS UNLIMTED

* Also at the Institute of Optics, University of Rochester

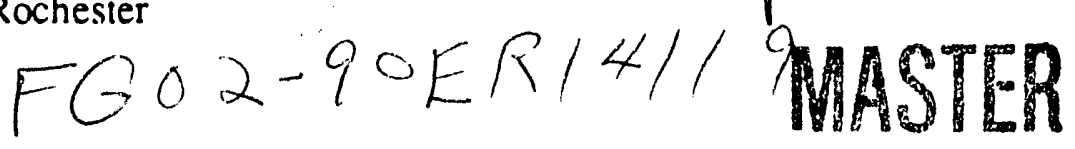


' sources and of the field not by these quantities but rather by their Fourier transforms, known as crossspectral densities:

$$
W_{Q}\left(r_{1}, r_{2}, v\right)=\int_{-\infty}^{\infty} \Gamma_{Q}\left(r_{1}, r_{2}, \tau\right) e^{2 \pi i v \tau} d \tau, \quad W_{V}\left(r_{1}, r_{2}, v\right)=\int_{-\infty}^{\infty} \Gamma_{V}\left(r_{1}, r_{2}, \tau\right) e^{2 \pi i v \tau} d \tau .
$$

Because the field variable and the source variable are related by the inhomogeneous wave equation, these two cross-spectral densities are also related. In particular, for radiation in free space one can show that ${ }^{2}$

$$
\left(\nabla_{1}^{2}+k^{2}\right)\left(\nabla_{2}^{2}+k^{2}\right) w_{v}\left(r_{1}, r_{2}, v\right)=(4 \pi)^{2} W_{Q}\left(r_{1}, r_{2}, v\right)
$$

Here $\nabla_{j}^{2}$ is the Laplacian operator acting with respect to the coordinates of the point $\mathbf{r}_{j},(j=1,2)$, and $1 k=2 \pi v / c, c$ being the speed of light in vacuo.

Two important quantities which are readily expressible in terms of the cross-spectral densities are of special interest. They are:

$$
\text { Spectral density: } \quad S_{A}(r, v)=W_{A}(r, r, v)
$$

Spectral degree of coherence: $\mu_{A}\left(r_{1}, r_{2}, v\right)=W_{A}\left(r_{1}, r_{2}, v\right) / \sqrt{S_{A}\left(r_{1}, v\right)} \sqrt{S_{A}\left(r_{2}, v\right)}$.

with $A=Q$ or $V$. One can show that the upper bound of the absolute value of the spectral degree of coherence is unity, i.e. that $0 \leq\left|\mu_{A}\left(r_{1}, r_{2}, v\right)\right| \leq 1$. The exireme value unity characterizes complete correlation, the other extreme value, zero, characterizes complete absence of correlation.

The researches which I will now describe have been largely concerned with elucidating how the spectral degree of coherence affects the nature of the radiated field, both in free space and in scattering rnedia. Because of limitations of time I will mainly consider radiation in free space. This may appear to be a severe restriction, but I hope to show you that there is still a lot to be learned even about this rather restricted case.

\section{COHERENCE EFFECTS IN RADIOMETRY}

Because of the complexity of studying energy transport on the basis of either Maxwell's equations

- or quantum electrodynamics, one frequently uses in the treatment of many wave-propagation problems, a much older and a more intuitive model, namely the theory of radiative energy transfer. The chief quantity in this theory is the so-called specific intensity of radiation, also known as the (spectral) radiance or the

1 (spectral) brighiness, $B_{v}(r, s)$. It is defined as the rate at which energy is radiated at frequency $\omega$, per unit area at $\mathbf{r}$ perpendicular to a unit vector $\mathbf{s}$, per unit solid angle around the s-direction. By heuristic arguments one can show that the radiance satisfies the iranspon equation

$$
s \cdot \nabla \cdot B_{v}(r, s)=-\alpha_{v}(r, s) B_{v}(r, s)+\int_{(4 \pi)} \beta_{v}\left(r, s, s^{\prime}\right) B_{v}\left(r, s^{\prime}\right) d \Omega^{\prime}+D_{v}(r, s),
$$

where $\alpha_{v}(r, s)$ is the extinction coefficient, $\beta_{v}\left(r, s, s^{\prime}\right)$ is differential scattering coefficient and $D_{v}(r, s)$ is the source function.

In spite of its extensive use, the conditions for the validity of this equation are not known and, in 1 fact, even the relationship between the radiance function and the basic quantities of Maxwell's 
electromagnetic theory are poorly understood. What is known is that the radiance function is not a measurable quantity under all circumstances. It should be really regarded as a kind of quasi-probability, analogous to the Wigner distribution function ${ }^{3}$.

Several definitions of the radiance function have been proposed on the basis of coherence theory. Here are two examples of proposed definitions of the radiance function of a planar source of any state of coherence in terms of the cross-spectral density function of the source 4.5 :

$$
\begin{aligned}
& B_{v}^{(1)}(r, s)=\left(\frac{k}{2 \pi}\right)^{2} s_{z} \int_{(z=0)} W\left(r+\frac{1}{2} r^{\prime}, r-\frac{1}{2} r^{\prime}, v\right) e^{-i k s \cdot r^{\prime}} d^{2} r^{\prime}, \\
& B_{v}^{(2)}(r, s)=\left(\frac{k}{2 \pi}\right)^{2} s_{z} \int_{(z=0)} W\left(r, r^{\prime}, v\right) e^{-i k s \cdot\left(r-r^{\prime}\right)} d^{2} r^{\prime} .
\end{aligned}
$$

The source is assumed to occupy a finite portion of the plane $z=0$ and $s_{z}$ is the component of the unit vector $s$ along the normal to the source plane.

The first definition, Eq. (7), is analogous to the quantum-mechanical definition of the Wigner distribution function. The second one, given by $\mathrm{Eq} .(8)$, is analogous to the phase-space distribution function associated with the so-called antinormal ordering. These quantities have some unphysical features. For example both $\mathrm{B}_{v}^{(1)}$ and $\mathrm{B}_{v}^{(2)}$ may become negative for some values of their arguments. They cannot, therefore, represent the radiance of radiative transfer theory, which postulates that the radiance is always associated with real energy flow and must, therefore, be always non-negative.

To obtain some insight into the relationship between radiative transfer theory and statistical wave theory we have first considered energy propagation in free space, which is the domain of traditional radiometry. In this case the equation of radiative transfer takes the simple form $\mathbf{s} \cdot \mathbf{B}_{\mathbf{v}}(\mathbf{r}, \mathbf{s})=0$ implying that, with s fixed, $B_{v}(r, s)=$ const, i.e. that $B_{v}(r, s)$ remains constant along each $s$-direction. Because geometrical concepts and results such as this, play a substantial role in radiometry, we have investigated whether it is possible to derive the radiometric model from coherence theory as a kind of geometrical optics limit, i.e. as a short wavelength limit or, more precisely, as an asymptotic limit as the

wave number $k=2 \pi / \lambda \rightarrow \infty$. We have found that this is, indeed possible for fields generated by a cerain class of sources, known as quasi-homogeneous sources ${ }^{6}$. A source of this class is characterized by the property that its spectral degree of coherence $\mu^{(0)}$ depends on its two spatial arguments $r_{1}$ and $r_{2}$ only through their difference and that its spectral density $S^{(0)}$ remains sensibly constant over distances of the order of the correlation length of the source. For such a source

$$
w^{(0)}\left(r_{1}, r_{2}, v\right)=\underbrace{s^{(0)}\left(\frac{r_{1}+r_{2}}{2}, v\right)}_{\begin{array}{c}
\text { spectral density } \\
\text { (slow function) }
\end{array}} \underbrace{\mu^{(0)}\left(r_{2}-r_{1}, v\right)}_{\begin{array}{c}
\text { spectral degree of } \\
\text { coherence } \\
\text { (fast function) }
\end{array}} .
$$

Many laboratory sources and many sources which occur in nature belong to this class. We will only consider planar sources of this kind.

By a long argument based on the solution of equation (3), we have obtained the following result ${ }^{7.8}$ : All the radiance functions of a quasi-homogeneous source, which can be defined as linear 
transform of the cross-spectral density, [which include those defined by Eqs. (7) and (8)], have the same asymptotic limit as $k \rightarrow \infty$, namely

$$
B_{v}(r, s)-k^{2} s_{z} s^{(0)}\left(r-\frac{z}{s_{z}} s_{\perp}, v\right) \tilde{\mu}^{(0)}\left(k s_{\perp}, v\right) \text {. }
$$

where $\vec{\mu}^{(0)}$ is the two-dimensional spatial Fourier transform of $\mu^{(0)}$. This result has the simple geometrical interpretation indicated in Fig. 1, which is in agreement with the equation for radiative

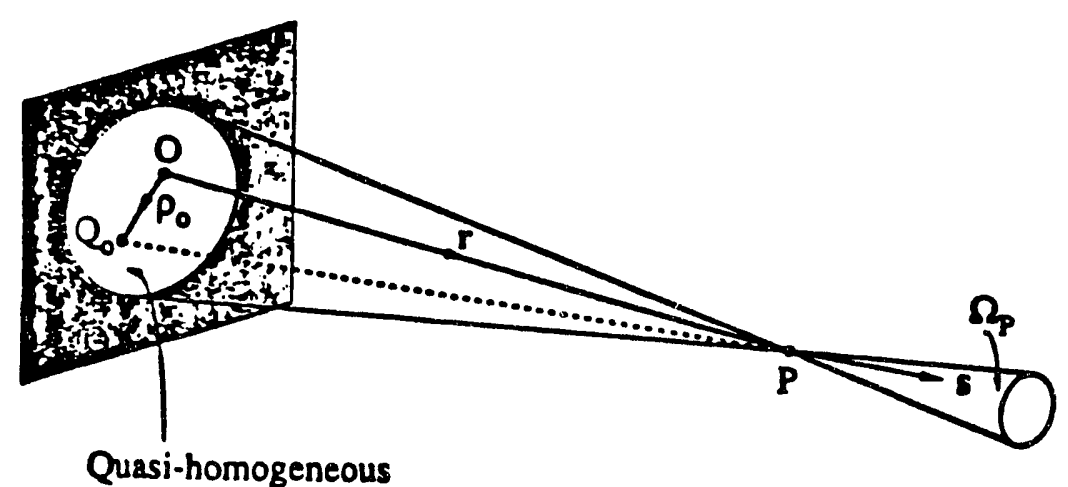

Fig. 1. Interpretation of Eq. (10) for the spectral radiance $\mathrm{B}_{\mathrm{v}}(\mathbf{r}, \mathbf{s})$ produced by a planar, secondary, quasi-homogeneous source $\sigma$. $P$ is a point whose location is specified by the position vector $r$.

$\mathrm{Q}_{0}$ is the point in the source plane whose position vector $p=r-\left(z / s_{2}\right) s_{\perp}$. It is the point of intersection with the source plane, of the line through the point $P$, in the direction of the unit vector s. [After J. T. Foley and E. Wolf, ref. 7].

energy transfer in free space. Moreover, one can show that expression (10) satisfies all the postulates of the phenomenological theory. We note that the radiance function given by the above formula depends not only on the spectral density of the source but also on its spectral degree of coherence. In fact the expression (10) for the radiance show's for the first time how the spectral degree of coherence of the source affects the radiance function of the field. Two examples are given in Figure 2. These results have a bearing on a number of problems of curreni research interest, for example in the field of non-imaging optics and in connection with synchroton radiation.

(a)

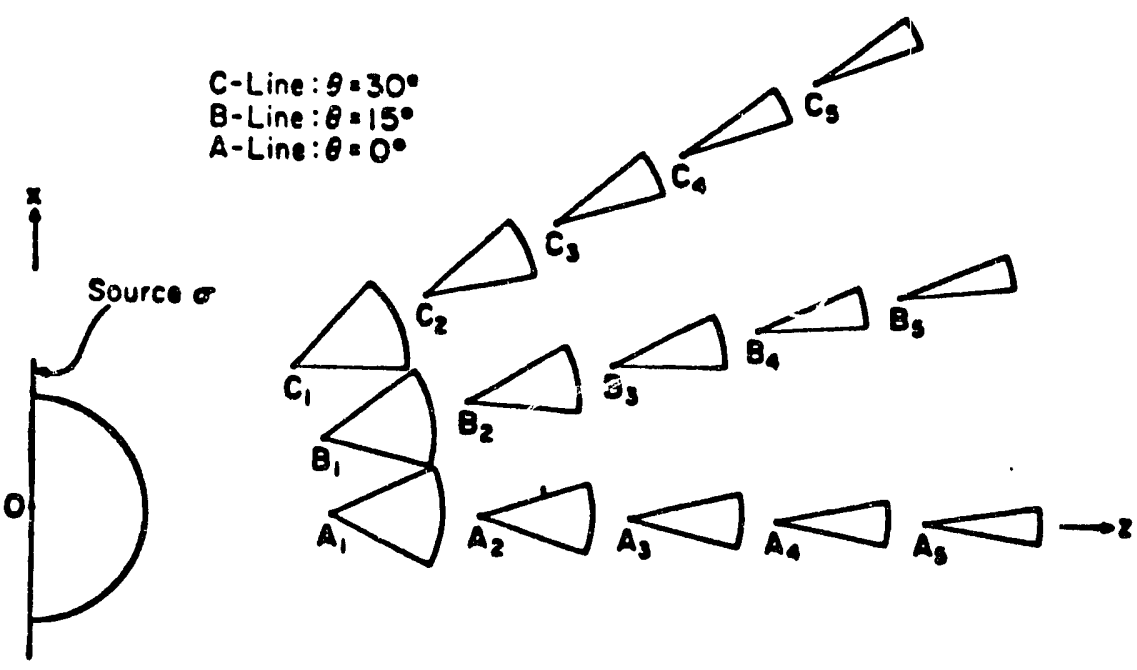


(b)

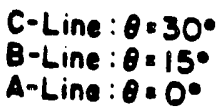

Fig. 2. Polar diagrams, calculated from Eq. (10), of the spectral radiance at different points in the $x, y$ plane, generated by some planar, secondary, quasi-homogeneous sources. The points with subscripts 1 , $2,3,4$ and 5 are at distances $r=4 \mathrm{~cm}, 6 \mathrm{~cm}, 8 \mathrm{~cm}, 10 \mathrm{~cm}$ and $12 \mathrm{~cm}$ respectively from the center) at the source. (a) From an uniform, circular, quasi-homogeneous, lambertian source, $\left\{\mu(0)\left(\mathbf{r}^{\prime}\right)=\left[\sin \left(k s^{\prime}\right) / k s^{\prime}\right]\right\}$, of radius $a=2 \mathrm{~cm}$. (b) From an uniform, circular, quasi-homogeneous, Gaussian-correlated source, $\left\{\mu^{(0)}\left(r^{\prime}\right)=\exp \left[\left(-r^{\prime 2} / 2 \sigma_{\mu}^{2}\right]\right\}\right.$, of radius $a=2 \mathrm{~cm}$ and with $\sigma_{\mu}=0.5 \lambda$ [After J.T. Foley and E. Wolf, ref. 7].

\section{COHERENCE EFFECTS IN SPECTROSCOPY}

I have spoken so far mainly about the effects which the coherence properties of a source have on the spatial distribution of the radiated energy. But w'e have found that the state of coherence of a source also affects the spectral distribution of the emitted radiation and this fact has lead to the discovery of a number of rather surprising new effects. In this connection let me remind you that it is an implicit assumption of all spectroscopy that the (normalized) spectrum of light which is incident on a detector after the light has propagated from its source through free space is the same, irrespective of the location of the detector and is equal to the spectrum at the source. I have shown that such an invariance of the spectrum of light on propagation is, however, not an universal propeny of light and that light produced by common laboratory sources is an exception in this respect. More specifically I have shown that the spectrum of light produced by a source depends not only on the source spectrum but also on the correlation between the source fluctuations at different source points ${ }^{9.11}$. This conclusion applies irespective of whether the source is primary, such as a set of radiating atoms, or is secondary, e.g. is the image of a primary source formed by an optical sysiem, or is a scattering medium illuminated by a beam of polychromatic light. This prediction has now been confirmed by experiments carried out in several different laboratories ${ }^{12}$.

1

The fact that, in general, the spectrum of light changes on propagation even in free space follows from the basic equation (3) which relates the cross-spectral densities of the field and of the source. If we wish to determine the spectrum $S_{v}(r, \omega) \equiv W_{v}(r, r ; \omega)$ of the field, we have to start from the crossspectral density of the source - not from its spectrum alone - and then determine the field spectrum by the following sequence, starting from Eq. (3): $W_{Q}\left(r_{1}, r_{2} ;(\omega) \rightarrow W_{V}\left(r_{1}, r_{2} ; \omega\right) \rightarrow W_{V}(\mathbf{r}, \mathbf{r} ; \omega) \equiv S_{V}(\mathbf{r}, \omega)\right.$.

Let me illustrate these remarks by a simple example ${ }^{13}$,involving two small fluctuating sources, 1 located in the neighborhood of points $P_{1}$ and $P_{2}$. Since the sources fluctuate, they must be described, for each frequency $\omega$, by statistical ensembles $\left\{Q\left(P_{1}, \omega\right)\right\}$ and $\left\{Q\left(P_{2}, \omega\right)\right\}$. The field which these sources I generate at a field point $P$ will also fluctuate. We may characterize the field fluctuations by a field 
ensemble $\{U(P, \omega)\}$. A typical realization of the field at point $P$, at frequency $\omega$, is given by the - formula

$$
U(P, \omega)=Q\left(P_{1}, \omega\right) \frac{e^{i k R_{1}}}{R_{1}}+Q\left(P_{2}, \omega\right) \frac{e^{i k R_{2}}}{R_{2}} .
$$

where $R_{1}$ and $R_{2}$ are the distances from $P_{1}$ and $P_{2}$ respectively to the point $P$. The spectrum of the field at the point $P$, is given by the expression

$$
S_{V}(P, \omega)=\left\langle U^{*}(P, \omega) U(P, \omega)\right\rangle \text {. }
$$

where the angular brackets denote the ensemble average. On substituting for $U(P, \omega)$ from Eq. (11) into Eq. (12) we find at once that

$$
S_{v}(P, \omega)=S_{Q}(\omega)\left[\frac{1}{R_{1}^{2}}+\frac{1}{R_{2}^{2}}\right]+\left[W_{Q}\left(P_{1}, P_{2}, \omega\right) \frac{e^{i k\left(R_{2}-R_{1}\right)}}{R_{1} R_{2}}+c c\right] \text {, }
$$

where cc denotes the complex conjugate. We have assumed here that the two sources have the same spectra, $S_{Q}(\omega)$. The crucial fact to note is that in addition to the source spectrum $S_{Q}(\omega)$ we have, according to Eq. (13), a contribution to the field spectrum which involves the function $W_{Q}\left(P_{1}, P_{2}, \omega\right)$ that characterizes the correlation of the fluctuations at the two source points. The formula shows that, in general, the spectrum of the field is not proporional to the spectrum of the source, but that it also depends on the correlation of the fluctuations of the tw'o small sources.

It is clear from Eq. (13) that in the special case when $W_{Q} \equiv 0$, i.e. when the two sources are completely uncorrelated or, as one says, they are mutually incoherent, the field spectrum will be proportional to the source spectrum. There are some other situations where that is also true, but the formula show's clearly, that in general, the field spectrum will differ from the source spectrum and that it may be different at different points in the field; and further, that the spectral changes depend on the nature of the correlation between the two sources.

The spectral changes induced by source correlations may be of many different kinds. In fact, depending on the nature of the correlations, the changes may be quite drastic including, for example, shifts of spectral lines, broadening or narrowing of lines and in some case even suppression of lines or generation of new ones. Some examples are shown in figures 3 and 4.

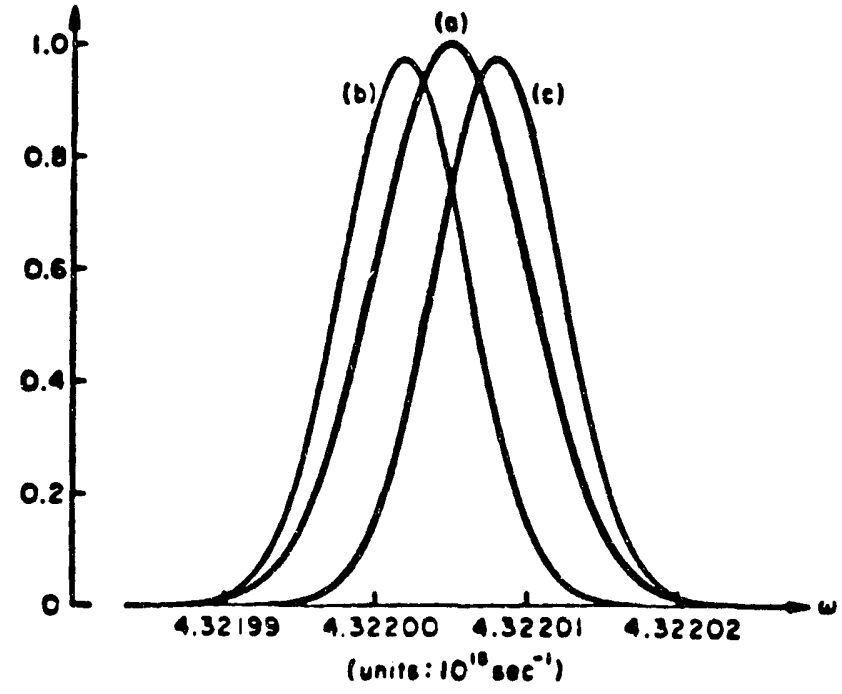

Fig. 3. Shifts of spectral lines induced by suitable correlations between two small sources: (a) the normalized spectrum $\mathrm{S}_{\mathrm{Q}}(\omega)$; $(b)$ and $(c)$ : the normalized spectra $S_{V}(\omega)$. IAfter $E$. Wolf, ref. 13]. 


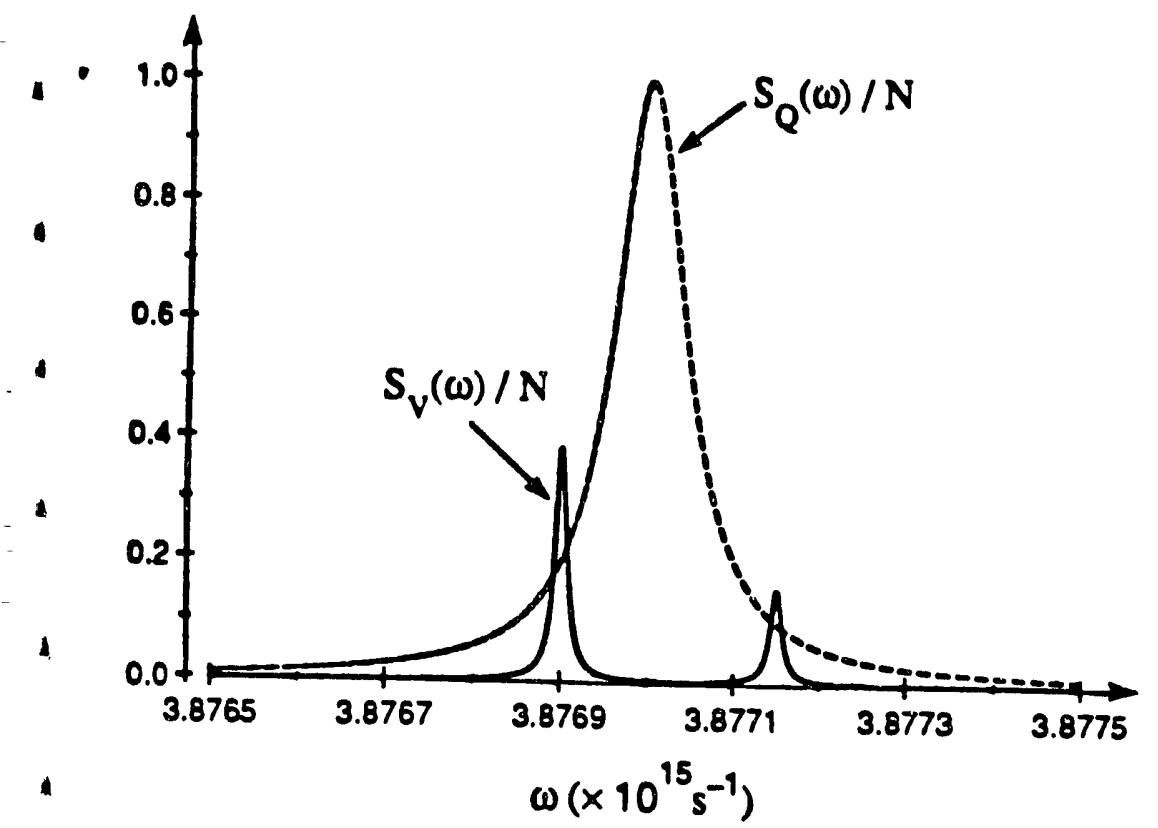

Fig. 4. Generation of two spectral lines, from a single spectral line of Lorentzian profile, with a suitable choice of the spectral degree of coherence. $N$ is a normalization constant. (cf. A. Gamliel and E. Wolf, ref. 14).

To bring out the essential feature of the phenomenon of correlation-induced spectral changes I have considered here only the simple case of two small correlated sources. However, this effect is very general and arises with two- or three-dimensional sources and also with some secondary sources, such as illuminated scattering media. Let me say a few' words about this.

We consider a medium whose refractive index or, equivalently, its dielectric susceptibility, vary randomly in space. Suppose that the medium is illuminated by a plane wave. Let us examine the scattered field in different directions. We can regard the illuminated medium as a source of radiation, because under the influence of the electric field, the bound electrons in the medium are displaced from their equilibrium positions and will oscillate. The polarization induced in the scattering medium in this way will, in general, be correlated over finite distances and will imitate correlations in primary or in secondary sources. Clearly the illuminated medium may be regarded as a secondary source. If one works out the theory in detail one finds ${ }^{15}$ that when the medium is statistically homogeneous, for example, the spectrum in the far field, $S_{V}^{(\infty)}(r, \omega)$, depends in the following way on the spectrum of the incident light $S_{V}^{(i)}(\omega)$ and on the

- correlation function $C_{\eta}(R, \omega)$ of the dielectric susceptibility, $\eta(r, \omega)$ :

$$
S_{v}^{(\infty)}(r u, \omega)=\frac{k^{4} v}{r^{2}} \dot{c}_{\eta}\left(k u-k u_{0}, \omega\right) S_{v}^{(i)}(\omega)
$$

Here

$$
\begin{aligned}
& \check{C}_{\eta}(K, \omega)=\frac{1}{(2 \pi)^{3}} \int C_{\eta}(R, \omega) e^{-i K \cdot R} d^{3} R, \\
& C_{\eta}(R, \omega)=\left\langle\eta^{*}(r, \omega) \eta(r+R, \omega)\right\rangle .
\end{aligned}
$$

$u_{0}$ and $u$ are real unit vectors in the directions of propagation of the incident and of the scattered field respectively and $\nu$ is the volume of the scattering medium. We may call this type of scattering static scattering, because nothing moves at the macroscopic level. It arises from the spatial inhomogeneities in the macroscopic properties of the medium.

The above formula for the far-zone spectrum shows that even in static scattering the spectrum of the scattered light differs, in general, from the spectrum of the incident light. Just like the examples we have already considered, this kind of scattering may also produce shifts of spectral lines, but the changes are necessarily rather small. In fact they cannot exceed the effective widths of the lines. However, much 
latger shifts can be produced in dynamic scattering, i.e. under circumstances when the macroscopic response of the medium varies sandomly not only in space but also in time. The underlying theory 16 is, - however, much more complicated.

A remarkable result that we have found in the course of our research regarding the effects of dynamic scattering on the spectrum of the scattered radiation is that when the space-time fluctuations in the dielectric susceptibility of the medium are suitably correlated, the induced spectral changes may completely imitate the Doppler effect, even though the source, the scattering medium and the observer are at rest with respect to each other. ${ }^{17.19}$ An example is given in Fig. 5.

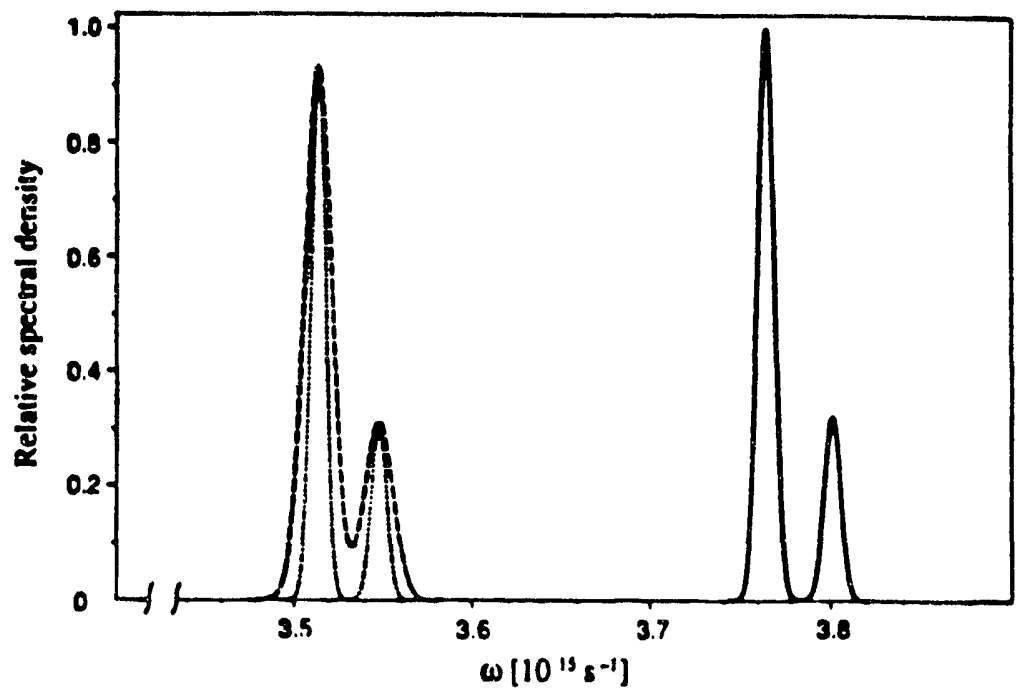

Fig. 5. Two 0 III lines $(\lambda=4959 \AA$ and $5007 \AA)$ as seen at rest (solid line), Doppler shifted (dotted line) and shifted by dynamic scattering, on a suitably correlated random medium (dashed lines). For details see $D$. $F$. V. James, $M$. Savedoff and $E$. Wolf, ref. 19, from which this figure is reproduced.

Various applications utilizing spectral changes which can be produced by source correlations have already been made and others are being considered. Unfortunately there is no time to discuss them here.

Let me conclude by saying that I hope to have shown you that correlation effects play a very significant role in many energy-related problems, particularly in connection with spatial and spectral distributions of energy' in radiation fields which are produced by sources of different states of coherence or which are generated by scattering on parially correlated random media.

\section{ACKNOWLEDGEMENT}

Much of the research described in this paper was supported by the Departmeni of Energy under grant DE-FG02-90ER 14119. The view's expressed in this article do not constitute an endorsement by the Deparment of Energy.

\section{REFERENCES}

1. M. Born and E. Wolf, Principles of Optics (Pergamon Press, Oxford, 6th ed., 1980), Sec. 10.2

1. 2. W. H. Carter and E. Wolf, J. Opt. Soc. Amer. 67, 785-796 (1977).

3. In this connection see E. Wolf, J. Opt. Soc. Amer. 68, 6-17 (1978).

4. A. Walther, J. Opt. Soc. Amer. 58, 1256 (1968).

5. A. Walther, J. Opt. Soc. Amer. 63, 1622 (1973).

16. W. H. Carter and E. Wolf, Opt. Acta 28, 227.244 (1981)

7. J. T. Foley and E. Wolf, J. Mod. Opt. 38, 2053-2058 (1991).

8. G. S. Agarwal, J. T. Foley and E. Wolf, Opt. Commun. 62, 67-72 (1987).

9. E. Wolf, Phy's. Rev. Lett. 56, 1370.1372 (1986).

10. E. Wolf, Nature 326, 363.365 (1987).

11. E. Wolf, Opt. Commun. 62, 12-16 (1987).

12. For a review of some of this work, see, for example, E. Wolf in International Trends in Optics, ed. J. W. Goodman (Academic Press, San Diego, 1991), pp. 221-232 and E. Wolf in 
Proc. Symp. Huygens' Principle 1690 - 1990: Theory and Applications, H. Blok, H.

13. E. Wolf, Phys. Rev. Lett. 58, 2646-2648 (1987).

14. A. Gamliel and E. Wolf, Opt. Commun. 65, $91-96$ (1988).

!

15. E. Wolf, J. T. Foley and F. Gori, J. Opt. Soc. Amer. A 6, $1142-1149$ (1989); ibid. A7, 173 (1990).

16. E. Wolf and J. T. Foley, Phys. Rev. A. 40, 579 -587 (1989).

17. E. Wolf, Phys. Rev. Letl. 63, 2220-2223 (1989).

1 18. D. F. V. James, M. Savedoff and E. Wolf, , Astrophys. J, 359, 67-71 (1990).

19. D. F. V. James and E. Wolf, Phys. Letts. A 146, 167-171 (1990).

\section{DISCLAIMER}

This report was prepared as an account of work sponsored by an agency of the United States Government. Neither the United States Government nor any agency thereof, nor any of their employees, makes any warranty, express or implied, or assumes any legal liability or responsibility for the accuracy, completeness, or usefulness of any information, apparatus, product, or process disclosed, or represents that its use would not infringe privately owned rights. Reference herein to any specific commercial product, process, or service by trade name, trademark, manufacturer, or otherwise does not necessarily constitute or imply its endorsement, recommendation, or favoring by the United States Goyernment or any agency thereof. The views and opinions of authors expressed herein do not necessarily state or reflect those of the United States Government or any agency thereof. 

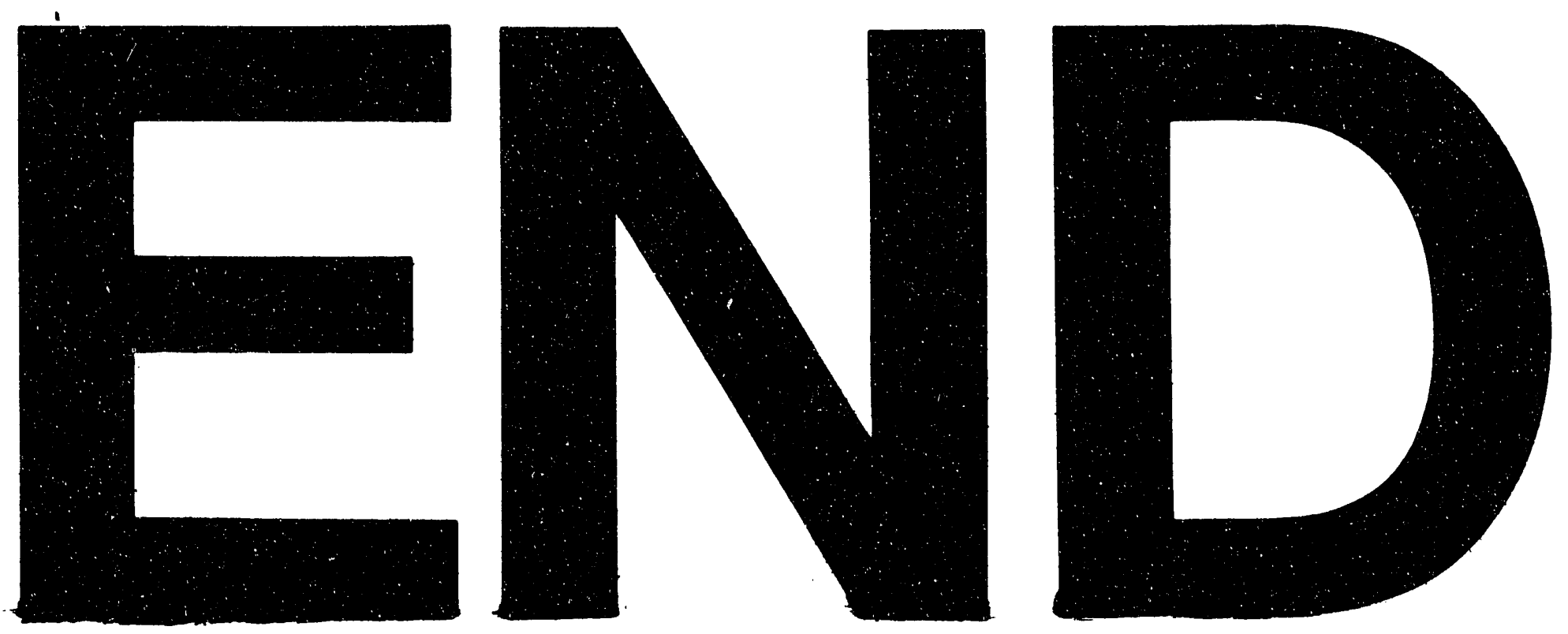

$=$
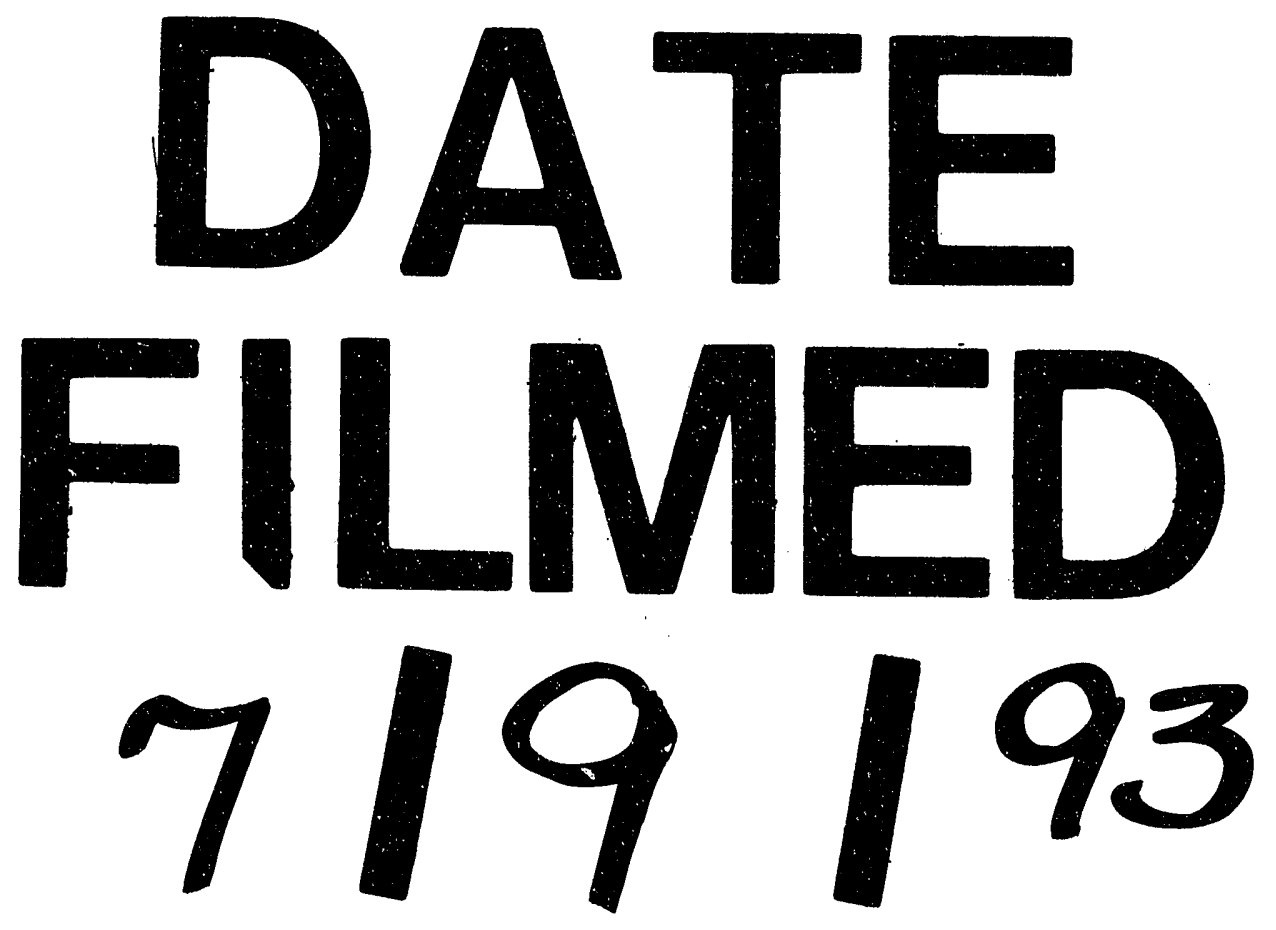


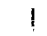

